\title{
FACTORES ASOCIADOS A LA PRÁCTICA DE PRUEBAS DE DETECCIÓN TEMPRANA EN CÁNCER DE MAMA.
}

Mendoza, $\mathrm{M}^{1}$, Rueda, $\mathrm{Y}^{2}$.

1. Mendoza Calderon Lourdes (2014), Bacterióloga, Especialista en Epidemiología, Instituto Nacional de Cancerología, Profesional Especializado, Bogotá, Colombia, marialourdesca@hotmail.com, mlcalderon@cancer.gov.co

2. Rueda Quitian Yolanda (2014), Bacterióloga, Especialista en Auditoría en Salud, Instituto Nacional de Cancerología ,Profesional Especializado, Bogotá, Colombia, yoyoruqui@gmail.com, yrueda@cancer.goc.co

\section{RESUMEN}

Objetivo: Revisar los factores asociados a la práctica de pruebas usadas para la Detección Temprana en Cáncer de Mama, como los son el Autoexamen, Examen Clínico de Mama y Mamografía. Métodos: Articulo de revisión. Resultados: Entre los estudios revisados se encontró que el conocimiento de las mujeres sobre el cáncer de mama, el grado de escolaridad, la edad y el lugar de residencia estaban asociados a la práctica del autoexamen. Con respecto a la práctica del examen clínico de mama, se observó además una asociación con la historia familiar de cáncer de mama. En relación a la mamografía además de los factores mencionados anteriormente, se evidenció que el tener pareja, la convivencia con menos de 4 personas, el consumo de 3 o más porciones de frutas y verduras y la presencia de comorbilidades como factores asociados a su práctica. Conclusiones: Los factores asociados a la práctica de estas pruebas son de carácter organizacional, sociodemográfico y relacionados con los estilos de vida. Los aspectos organizacionales y relacionados con los estilos de vida, como la dieta, el conocimiento frente al cáncer de mama y las barreras administrativas son modificables, mientras que los factores sociodemográficos no lo son tanto como la edad, el nivel educativo, el estado civil y el estrato socioeconómico. La educación desde todos los ámbitos es la mejor estrategia para disminuir las barreras que impiden la práctica de pruebas para la detección temprana del cáncer de mama.

Palabras clave: neoplasias de la mama, cribado, detección precoz del cáncer

\section{FACTORS ASSOCIATED WITH TESTING PRACTICE IN EARLY BREAST CANCER}

\section{ABSTRAC}

Objective: To review the factors associated with the practice of tests used for Early Breast Cancer Detection, as are the self-exam, clinical breast exam and mammography. Methods: Review Article. Results: Among the studies reviewed knowledge about breast cancer was found, the schooling age and place of residence were associated with the practice of self-examination 
with respect to the practice of clinical breast examination, was also found a association with family history of breast cancer, and in relation to mammography in addition to the factors mentioned above, have revealed the couple, living with less than 4 people, consuming 3 or more servings of fruits and vegetables and presence of comorbidities associated with practical factors.

Conclusions: Factors associated with the practice of these tests are organizational, related sociodemographic and lifestyle character. Organizational and related lifestyles, such as diet, awareness against breast cancer and administrative barriers factors are modifiable, while sociodemographic factors are not so much as age, educational level, marital status and socioeconomic status. Education on all fronts is the best strategy to reduce barriers to the practice tests for the early detection of breast cancer.

Key words: breast neoplasms, early detection of cancer, cancer screening tests

\section{INTRODUCCION}

El cáncer de mama representa el segundo cáncer más común en el mundo, las tasas de incidencia varian casi 4 veces a través de las regiones del mundo con tasas que van desde 27 por 100.000 en África y Asia del este a 96 por 100.000 en Europa Occidental. El rango de las tasas de mortalidad entre las regiones del mundo es menor que el de incidencia, con tasas que van del 6 por 100.000 en Asia Oriental a 20 por 100.000 en África Occidental. (Ferlay ,2012).

En Colombia el cáncer de mama se presenta como un problema creciente de Salud Pública, actualmente constituye la principal localización y causa de muerte por cáncer en las mujeres, con tasas de incidencia alrededor de 35.7 por 100.000 mujeres, y tasas de mortalidad cerca de 10,8 por 100.000. (Ferlay ,2012).El cáncer de mama ocupó el tercer lugar en la mortalidad por cáncer en mujeres, después del cáncer de cuello de útero y de estómago. Este representa cerca del 14\% de las muertes por cáncer en las mujeres colombianas. (Piñeros, 2010, p.43).

En relación con la mortalidad por cáncer de mama en Colombia, Bogotá fue la única zona con una tendencia significativa al descenso, en relación a departamentos como Tolima y Magdalena y aunque esta fue muy leve $(0,09 \%)$ de acuerdo a cifras publicadas en el Atlas de Mortalidad por Cáncer en Colombia 2010, esto podría atribuirse al mayor acceso a tratamientos adyuvantes de buena calidad, en combinación con una mayor frecuencia de realización de mamografías de tamización. La tendencia de la mortalidad a estabilizarse durante el último periodo (2000-2006) llama la atención, y bien puede estar indicando una mejoría en el pronóstico de este tipo de cáncer.(Piñeros, 2010, p.43).

Sin embargo estos datos fueron extraídos de publicaciones del año 2010, en el que la tasa de mortalidad para el país estaban alrededor de 9,2 por 100.000 mujeres, pero de acuerdo a publicaciones del 2012 está aumentó a 10,8 por 100.000 , lo que evidencia un incremento en la mortalidad por cáncer de mama. (Ferlay ,2012)(Piñeros, 2010). Esto puede estar indicando serios problemas en el diagnostico precoz de esta enfermedad, así como también la persistencia de diferentes barreras para acceso al tratamiento oportuno, no obstante existe un factor que se debe tener en cuenta al momento de estimar este incremento en la mortalidad y es la práctica por parte de la población de las pruebas que han sido evaluadas de manera positiva en beneficio de la reducción de la mortalidad por cáncer de mama. 
Este artículo tiene como objetivo hacer una revisión de los factores asociados con la práctica de pruebas usadas en detección temprana de cáncer de mama, como son el autoexamen, el examen clínico de mama y la mamografía, revisando aspectos generales de cada una de estas pruebas y su práctica en la población.

\section{Conceptos relacionados con pruebas usadas en detección temprana en cáncer de mama.}

La detección temprana se considera como la identificación de una enfermedad, en este caso, el cáncer de mama, en un punto de su historia natural cuando puede ser tratada con técnicas que tendrán menor impacto físico y mayor probabilidad de curación. Existen tres estrategias claramente definidas para la detección temprana en cáncer de mama: la detección temprana sin tamizaje, el tamizaje de oportunidad y el tamizaje organizado. La detección temprana sin tamizaje está basada en la educación tanto de la población como del personal de la salud con el fin de identificar signos y síntomas que conlleven a un diagnóstico temprano. El tamizaje como tal involucra la realización de pruebas en individuos sanos con el fin de identificar la población en riesgo. Este puede ser de oportunidad cuando el uso de estas pruebas no está dentro de un programa formal; u organizado cuando existe un programa formal de tamizaje en el que deben cumplirse ciertas normas técnicas y administrativas. (Anderson, 2003).

Las pruebas utilizadas para el tamizaje en cáncer de mama son el autoexamen, el examen clínico de la mama y la mamografía. Existe controversia en relación a la evidencia a nivel mundial con respecto a la reducción de la mortalidad de cada una de estas pruebas, sin embargo diferentes estudios alrededor del mundo aportan cierta evidencia que apoyan el uso de estas pruebas de manera combinada en beneficio de la reducción de la mortalidad para cáncer de mama.

En Colombia la Resolución 412 del 2000, la cual establece las actividades $y$ procedimientos para el desarrollo de protección específica y detección temprana para enfermedades de interés en salud pública, conviene la práctica de la mamografía( de 4 proyecciones) cada 2 años por encima de los 50 años de edad, independientemente de la presencia o no de signos o síntomas en la mama, También establece que toda mujer con mamografía sospechosa, debe tener acceso a todos los servicios de diagnóstico definitivo mediante biopsia por aspiración con aguja fina (BACAF) y al tratamiento consecuente.

La resolución 3384 2000, fija las metas de cumplimiento establecidas para el régimen contributivo del $20 \%$, mientras que para los demás regímenes no establece coberturas exigibles, y además adiciona a la detección temprana actividades de toma y lectura de biopsia.

Esta normatividad, evidenció algunas falencias, como el hecho de exigir coberturas tan bajas, el no tener una visión clara acerca de la población objeto y no establecer coberturas exigibles para los otros regímenes.

Sin embargo, por medio del Acuerdo 029 de 2011, se incluyeron las acciones de detección temprana del cáncer de mama en el régimen Subsidiado, de la misma forma que para el Contributivo.

En el año 2006 el Instituto Nacional de Cancerología, desarrolló unas recomendaciones para la detección temprana de cáncer de mama en Colombia. El documento propone la organización de estrategias de detección temprana para mujeres sintomáticas independientemente de la edad, y la tamización de base poblacional para mujeres asintomáticas entre los 50 y 69 años de edad con mamografía bienal y examen clínico anual. 
Con la expedición de la llamada Ley Sandra Ceballos ley 1384 de 2010 por la cual se establecen las acciones para la atención integral del cáncer en Colombia se espera entre otros logros, impactar algunas de las metas propuestas en el Plan Decenal para el Control del Cáncer en Colombia 20122021, como son el incremento de los casos de cáncer de mama en estadios tempranos al $60 \%$ antes del 2021 , incrementar antes del 2021 la cobertura de mamografía de tamización bianual al $70 \%$ en mujeres de 50-69 años y garantizar el acceso oportuno a confirmación diagnóstica y tratamiento al $100 \%$ de las mujeres con mamografía reportada BIRADS 4 o más.

El acceso y oportunidad a pruebas de detección temprana esta mediado por diferentes factores desde el ámbito sociodemográfico, el ámbito personal y el ámbito administrativo. Estos diferentes factores están a su vez mediados por algunos determinantes dados por los cambios sociales de las últimas décadas, como el aumento de la población y la esperanza de vida, la industrialización de las áreas rurales, las grandes migraciones, la exposición a carcinógenos ambientales, el acceso a los servicios de salud y los factores de riesgo para cáncer.

\section{Factores asociados a la práctica del autoexamen}

El autoexamen de mama es un procedimiento realizado por la mujer para examinarse física y visualmente detectando cualquier cambio en sus senos y axilas. (Semiglazov VF, 1993).

En relación con el autoexamen dos ensayos clínicos aleatorizados en (Shangai) (Thomas $D, 2002$ ) y uno en San Petesburgo (Rusia) (Semiglazov VF, 1993) no se demostró ninguna reducción en el riesgo de morir por cáncer de mama, por el contrario se demostró un incremento en el número de biopsias innecesarias. Dos revisiones sistemáticas realizadas sobre autoexamen han encontrado información primaria deficiente colmada de sesgos y sin diferencias significativas en la reducción de la mortalidad entre las mujeres que se practicaron el autoexamen y aquellas que no se lo practicaron (RR: 1,05; IC 95\% 0,91,24). (Kosters JP, 2004).

Basados en este tipo de evidencia se concluyó que el autoexamen no disminuye la mortalidad por cáncer de mama, por lo cual no se recomienda como una prueba única de tamización y se debe complementar con otras pruebas como el examen clínico de mama y la mamografía.

Un estudio cuyo objetivo era determinar los factores asociados con una ejecución correcta del autoexamen de mama en mujeres adultas residentes en la ciudad de Tunja Boyacá, mostró que la prevalencia de realización del autoexamen de mama fue de apenas el $27,8 \%$ y de estas solo el $6.2 \%$ lo realizaban de manera adecuada. Los principales factores asociados con la realización adecuada del autoexamen de mama fueron: haber aprendido $y$ mecanizado una técnica regular $y$ sistemática, la escolaridad secundaria 0 mayor, y tener conocimiento sobre el cáncer de mama.(Manrique F, 2012).

Cifras similares en la práctica de autoexamen de mama fueron reportadas en países como Cuba y Perú. Un estudio realizado en la comunidad de Santos Suarez en Cuba con el objetivo de determinar la incidencia de cáncer de mama y la utilización de métodos de detección precoz en mujeres mayores de 20 años mostró que el $36 \%$ de las mujeres encuestadas no se practican el autoexamen de mama.(Prendes Marianela, 1998).Un estudio similar que buscaba conocer las conductas preventivas para el cáncer de mama en mujeres mayores de 20 años en la ciudad de Sullana mediante técnicas de auto examen de mama y mamografías; encontró que el $57 \%$ de las mujeres nunca se realizó un autoexamen de mama, sin embargo el $56 \%$ refiere conocer la utilidad 
del autoexamen de mama, el $43 \%$ de las mujeres encuestadas conocen la técnica pero solo un $8 \%$ lo practican correctamente.(Villacrés K, 2002).

Situación diferente es la reportada en países como Brasil y México. Con el objetivo de verificar el conocimiento de las mujeres acerca del autoexamen de mama y su importancia en la detección precoz del cáncer, así como analizar su práctica e identificar los motivos de su no realización; se realizó en tres unidades de salud de Ceará (Brasil), un estudio el cual mostró que el $55 \%$ de las mujeres encuestadas consideró hacerse el autoexamen en algún momento, el otro $45 \%$ que dijo no habérselo practicado refirió motivos como el desconocimiento y el olvido.(Carvalho $A$, 2006). Una revisión de los resultados de la Encuesta Nacional de Salud Reproductiva de México mostró que el $74 \%$ de las mujeres encuestadas examina sus mamas y los principales factores asociados a esta práctica fueron la edad, el lugar de residencia la escolaridad y el nivel socioeconómico. El $50 \%$ de las mujeres que revisan sus mamas lo hacen más de una vez al mes y solo el $10 \%$ dijo hacerlo una vez al mes; entre los principales motivos para no practicarse este examen estuvieron no considerar que sea una práctica importante, y no saber cómo realizarla.(López L, 2009).

Un estudio transversal realizado en una población de Jordania, que buscaba evidenciar los factores y creencias que pueden estar relacionados con la práctica del autoexamen de seno, evidenció que la motivación, la sensibilidad fueron variables que mostraron asociación positiva con la práctica del examen en el año anterior. Mientras que los beneficios que aporta el examen, la susceptibilidad y motivación influyeron en la intención de realizar el autoexamen de mama a futuro. La edad de la mujer, el nivel de educación, después de haber escuchado o leído acerca los tumores de mama, y la historia personal de los tumores de mama fueron también resultados ser predictores significativos de la práctica del autoexamen de seno. (Wasileh Petro-Nustus, 2002).

En Colombia de acuerdo a la encuesta de demografía y salud del año 2010 (ENDS), el $90 \%$ de las mujeres mencionó conocer el autoexamen de mama, el $62 \%$ de las mujeres encuestadas manifestó haberse practicado el autoexamen de mama cifra que se incrementó con respecto a lo reportado en la ENDS 2005 25\%. Y entre los factores asociados a esa práctica tenemos mujeres con mayor nivel educativo, residente en zonas urbanas y con un mayor índice de riqueza. El 39\% de las mujeres que declararon hacerse el autoexamen, lo hacen cada mes, el 10\% dicen hacérselo a diario, $9 \%$ lo hace cada seis meses, $6 \%$ lo hace semanalmente y $5 \%$ lo ha hecho sólo una vez. El $24 \%$ de las mujeres manifestó otra frecuencia de realización. La mayor proporción de mujeres que declararon hacérselo a diario fueron mujeres del menor nivel educativo18\%.

El autoexamen como prueba única de tamización no está relacionado directamente con la reducción de la mortalidad por cáncer de mama, además este debe realizarse de manera correcta y con una periodicidad adecuada, para lo cual se hace necesaria la educación por parte del personal de salud mediante la incorporación de campañas que incentiven el autocuidado por parte de las usuarias de los sistemas de salud.

\section{Factores asociados a la práctica del examen clínico de mama}

Un examen clínico de mama (ECM) es un examen mediante el cual personal entrenado, palpan la mama para detectar la posible presencia de bultos $u$ otras alteraciones.(IARC, 2002).

Una publicación en el año 2003 encontró que la utilización de una estrategia menos 
sensible que la mamografía, como el examen clínico de mama, podría conferir una reducción en la mortalidad por cáncer de mama en $11 \%$, equivalente a la mitad de la observada con mamografía.(NS. W. 2003). Otro ensayo clínico realizado en 2011 en el distrito de Trivandun en India, cuyo objetivo fue evaluar si tres rondas de examen clínico de mama cada tres años tendrían efecto en la reducción de la incidencia y mortalidad por cáncer de mama; mostró en resultados preliminares tasas de incidencia estandarizadas por edad, después de la primera ronda de tamización para estadios tempranos de 18,8 y 8,1 por 100,000 mujeres, en los grupos de intervención y control respectivamente y en estadios avanzados 19,6 y 21,7 por 100,000 mujeres, en los grupos de intervención y control.(Sankaranarayanan R. 2011). Estos y otros estudios coinciden en el beneficio limitado del examen clínico de mama en la reducción de la mortalidad, como prueba única de tamización pero de utilidad diagnostica que podría contribuir a la detección temprana en sitios con alta incidencia y presentaciones avanzadas y donde los recursos para la mamografía sean insuficientes. Aunque el examen clínico es incapaz de detectar lesiones pequeñas que pueden ser solo vista a través de la mamografía, podría potencialmente mejorar la situación donde la mayoría de cánceres se detecta en estadios III Y IV.(Díaz S, 2005, p. 98).

Kardinah y colaboradores, encontraron en un grupo de mujeres en Indonesia una efectividad similar del ECM, a la encontrada con la mamografía en el diagnóstico de canceres prevalentes en población no tamizada previamente, esto refuerza cada vez más la teoría de la utilidad del ECM en países de bajos y medianos ingresos. (Kardinah, 2014). Otra publicación que apoya dicha hipótesis fue un estudio Egipcio que aleatorizó 14.807 mujeres con ECM en un grupo de intervención y un grupo control y cuyos resultados iniciales demostraron que la distribución de los estadios al momento del diagnóstico fue mejor en el grupo de intervención con respecto al grupo control. (Miller $A B, 2008$ ).

Un estudio publicado en el 2009 en mujeres de 50 a 69 años de edad, en el que se pretendía determinar la cobertura de examen clínico de mama y mamografía de tamización para cáncer de mama en un grupo de mujeres bogotanas afiliadas a servicios de salud, concluyó que el 59,3\% de las mujeres encuestadas se había realizado el examen clínico de la mama en algún momento de la vida y el $65,6 \%$ de estos exámenes fueron realizados con fines de tamización. Entre los factores asociados positivamente con la práctica del examen clínico de mama se mencionaron el nivel educativo mostrando la formación universitaria una relación consistente con la práctica de este examen, además de la historia familiar de cáncer de mama. La fortaleza de este estudio frente a muchos otros que abordan el tema, es que hace diferencia entre los exámenes de tamización y diagnósticos lo que permite entender un poco más acerca de la adherencia a este tipo de programas de detección temprana en cáncer de mama. (Arboleda W. 2009).

Otra investigación con un objetivo similar al estudio mencionado anteriormente, en mujeres del mismo rango de edad pero afiliadas al régimen subsidiado en la ciudad de Manizales, mostró que la frecuencia de vida de uso del examen clínico fue de $53 \%$ de los cuales el $3 \%$ fue diagnosticado con cáncer de mama, este estudio también mostró que un $53 \%$ tenía ECM de tamización en el último año, resultado por encima de lo reportado para el país (46.7\%).A diferencia del estudio anterior este no mostró ninguna asociación con la práctica del ECM, quizá porque uno de los factores que se han venido reportando como asociados a esta práctica es el nivel educativo, en particular la formación universitaria, y en este estudio predominó la baja escolaridad en las mujeres 
encuestadas (Arboleda W, 2012), (Arboleda W. 2009).

La Encuesta Nacional de Salud Reproductiva de México, reportó la edad, el lugar de residencia, la escolaridad y el estrato socioeconómico asociado significativamente a la práctica y frecuencia del examen clínico de la mama, reforzando esto, la teoría de la importancia que reviste la educación en este tipo de prácticas. Las mujeres con estudios superiores se someten a este examen con el doble de frecuencia a que aquellas de baja escolaridad $\quad(\mathrm{RM}=2.2 ; \quad \mathrm{IC} 95 \%$ : 1.6 3.1).(López L, 2009).

Para la práctica del examen clínico de mama, el último reporte de la encuesta Nacional de Demografía y salud de Profamilia 2010, mostró cifras muy cercanas a las antes reportadas, $47 \%$ para la frecuencia de uso en la vida de ECM, de estas el $52 \%$ pertenecian al rango de edad entre 50-69 años, el 58\% fueron mujeres con educación superior, y el $65 \%$ se realizaron el examen con fines de tamización, la frecuencia de examen clínico de mama por tamización se incrementa con la edad, con el nivel educativo, con el índice de riqueza y entre aquéllas que residen en la zona urbana. El $47 \%$ de la mujeres manifestó haberse realizado el ECM en el último año muy acorde lo establecido a las recomendaciones del Instituto Nacional de Cancerología en el 2006.

\section{Factores asociados a la práctica de la mamografía}

La mamografía es un tipo de imagen de las glándulas mamarias para detectar el cáncer de seno en un estado temprano utilizando una baja dosis de rayos $X$, en donde se toman distintas proyecciones para examinar las glándulas mamarias detectando nódulos benignos o malignos. Aunque la dosis de radiación ionizante es baja se considera cancerígena, así que la solicitud de este examen debe incluir la evaluación del riesgo/beneficio para las mujeres que se practiquen este examen. Los resultados de la mamografía se deben informar en un sistema BIRADS (sistema de informes y registros de datos de imagen de la mama), cuyo objetivo es minimizar la variabilidad en la emisión de los informes de las mamografías.(Manual Para La Detección del Cáncer de Mama, 2012).

Durante los años 70 y 80 diferentes ensayos clínicos reportaron la utilidad de la tamización organizada con mamografía en la reducción de la mortalidad por cáncer de mama en países desarrollados, entre estos podemos mencionar el Healthlnsurance Plan o estudio HIP (Shapiro S, 1997), el estudio de Malmo (Andersson I JL, 1997), el de dos Condados (Tabar L, 2000),el estudio de Edimburgo (Alexander FE, 1999), y el estudio de Estocolmo (Frisell J, 1997). Estos estudios tuvieron algunas fallas metodológicas que fueron evidenciadas más adelante.

Aunque el metanálisis de la US TaskForce encontró una reducción en la mortalidad de 0,84 (IC 95\% 0,77-0,91) para todos los grupos de edad y la IARC encontró un riesgo relativo de 0,75 (IC 95\% 0,67-0,85) en la mortalidad por cáncer de mama con el uso de tamización con mamografía en la mujeres de 50 a 69 años de edad (Humphrey LL, 2002), (IARC, 2002). Un estudio publicado por (Gotzche\& Olsen, 2004) no mostro que la tamización con mamografía produjera una reducción significativa de la mortalidad por cáncer de mama 0,97 (IC 95\% 0,82-1,14) a trece años de seguimiento, resultados atribuibles a errores en obtención de la información primaria.

Después de 3 décadas de seguimiento el estudio de dos condados Suecia, logro demostrar un reducción altamente significativa en la mortalidad por cáncer de mama con el uso de la mamografía $R R=0.69$ (IC 95\%0,56, 0,84). (Tabar L, 2011). 
Recientemente causo controversia una publicación Canadiense, que no encontró reducción en la mortalidad con el uso de mamografía anual en mujeres de 40-59 años, después de 25 años de seguimiento más allá de la ofrecida por el ECM.(Miller $\mathrm{A}$, 2014).

A pesar de los inconvenientes presentados por los estudios anteriores y las críticas recibidas, no se ha logrado restar evidencia a la disminución en la mortalidad del cáncer de mama, con el uso de la mamografía. Basados en la recomendación de la IARC, en la que se concluye sobre la efectividad de la mamografía en la reducción de la mortalidad por cáncer de mama en mujeres de 50 a 69 años, con un resultado global entre $0 \%$ y $25 \% \quad$ (OR 1 a 0,75 , respectivamente). De acuerdo a estas observaciones en Colombia se establecieron guías, protocolos y normas dirigidas al uso de la mamografía como prueba de tamización útil en la reducción de la mortalidad por cáncer de mama, teniendo en cuenta la edad y la periodicidad con que se realice.

La guía de práctica clínica 2013 para la detección temprana, tratamiento integral, seguimiento y rehabilitación del cáncer de mama publicada por el Ministerio de Salud recomienda realizar tamización de base poblacional organizada mediante mamografía de dos proyecciones, cada dos años en mujeres de 50 a 69 años de edad, siempre incluido dentro de un programa de detección, diagnóstico y tratamiento oportuno del cáncer de mama. No se recomienda realizar tamización de rutina con mamografía en mujeres de 40-49 años de edad. La decisión de iniciar tamización regular con mamografía cada dos años antes de los 50 años debe ser individual y debe tener en cuenta el contexto del paciente incluyendo sus valores en relación con los beneficios. Se recomienda realizar detección temprana en mujeres sintomáticas independientemente de su edad, utilizando las estrategias diagnósticas adecuadas que pueden incluir la mamografía o la ecografía.
En el año 2013 se publicó un estudio que buscaba determinar los factores asociados con la práctica de la mamografía en mujeres mayores de 50 años en la ciudad de Cartagena (Colombia), y que mostró que el $60 \%$ dijo haberse practicado alguna vez en la vida la mamografía, pero solo el $34,5 \%$ manifestó habérsela practicado en los últimos 2 años tal como lo establece la guía y las recomendaciones dispuestas. Entre los factores que explican el uso de la mamografía encontramos tener pareja, convivir con menos de 4 personas, y tener un nivel superior de escolaridad.(Ávila I, 2013).

En el estudio publicado por Arboleda y col, en la Ciudad de Bogotá en el 2009, se reportaron cifras un poco más altas con respecto al uso de la mamografía en algún momento de la vida en mujeres de 50 a 69 años de edad $79,8 \%$, pero solo el 65 , $\%$ manifestó habérsela practicado con fines diagnósticos, casi más del $50 \%$ de lo reportado en la Ciudad de Cartagena. Al ajustar por edad en las mujeres entre 60-69 años, el nivel educativo y la historia familiar de cáncer de mama estuvieron asociados positivamente a la práctica de tamización para cáncer de mama.

Al revisar un estudio de casos y controles llevado a cabo en España y que tenía como objetivo estimar la prevalencia de prácticas de cribado de cáncer y evaluar su asociación con los estilos de vida, encontramos que el $85 \%$ de los sujetos se habían realizado alguna prueba de cribado (mamografía, citología, sangre oculta en heces, colonoscopia/ sigmoidoscopia, 0 antígeno prostático), en los últimos 5 años, porcentaje que fue de un $75 \%$ en los hombres y un $93 \%$ en las mujeres. La mamografía presento una elevada prevalencia $98 \%$ en mujeres de 50-69 años. El uso de la mamografía estuvo asociado con las mujeres que consumían 30 más porciones de frutas y verduras al día $(\mathrm{P}<0,05)$. La participación en las pruebas de cribado siempre fue mayor entre los sujetos 
que presentaban comorbilidades, excepto la citología. Ninguna práctica de cribado se asoció con antecedentes familiares de cáncer. $Y$ en cuanto a la relación de las pruebas de cribado entre sí solo se encontró relación entre la mamografía y la citología. (Perea M Dolores 2012).

La EDNS 2010, reporta coberturas de mamografía muy bajas para mujeres en el rango de edad de 50-69 años 49\%. Y de estas el $73 \%$ dijo haberse practicado el examen con fines de tamización, cifra superior con respecto a la ENDS 2005 que fue de $30,4 \%$. La proporción de las mujeres que se han hecho la mamografía aumenta con la edad, el nivel educativo y el índice de riqueza. Así, mientras que en las mujeres sin educación el $17 \%$ se había realizado una mamografía, entre aquéllas con un nivel de educación superior, la proporción fue de $59 \%$.

Con el uso de tecnologías de imágenes emergentes, como la tomosintesis de mama digital se espera transformar la detección temprana de cáncer de mama, Sin embargo la adopción de estas nuevas tecnologías en la práctica clínica es aún un desafío tanto en la evidencia de la clínica como en la relación de costo-efectividad.(Lee IC,2013)

\section{DISCUSIÓN}

Muchos de los factores asociados a la práctica de este tipo de pruebas son de carácter sociodemográfico, organizacional y personal relacionado con los estilos de vida. Algunos de los factores asociados a la práctica de pruebas de detección temprana para cáncer de mama son modificables como los organizacionales y personales relacionados con los estilos de vida, otros no los son tanto como los sociodemográficos.

Las características sociodemográficas asociadas a la participación de estas pruebas están relativamente establecidas, y se ha encontrado una asociación positiva con mayor edad, nivel de educación más alto, estar casado y mayor nivel socioeconómico.(Martín-López R, 2010).
La edad es un factor decisivo para la práctica de pruebas de detección temprana, más si se tiene en cuenta que existe un establecimiento de la edad de inicio de realización de este tipo de pruebas. De acuerdo a la guía de práctica clínica para la detección temprana, tratamiento integral, seguimiento y rehabilitación del cáncer de mama 2013, se recomienda la realización del examen clínico de la mama a partir de los 40 años, como parte del examen clínico general de la mujer, por lo menos una vez al año. Esto acompañado de la implementación de escenarios para la enseñanza del examen clínico de la mama, con el fin de generalizar y estandarizar la técnica. La guía no recomienda la realización del autoexamen de la mama como estrategia de tamización. Se invita a la enseñanza del autoexamen como estrategia de concientización y autoconocimiento. La mamografía es recomendada para las mujeres entre los 50 69 años.

Para la práctica de mamografía y examen clínico de mama la ENDS 2010 reporta cifras alrededor del $50 \%$ para mujeres en el rango de edad de 50-69 años, aun cuando la recomendación para el ECM es a partir de los 40 años.

El nivel educativo incide de manera significativa en la probabilidad de realización de las tres pruebas de detección temprana para cáncer de mama, sobre todo en mujeres con estudios universitarios. Las mujeres de baja escolaridad tienen una menor probabilidad de someterse a este tipo de pruebas razón por la cual presentan un mayor riesgo de llegar a un cáncer de mama en estados avanzados. Aun cuando los sistemas de salud no tienen la responsabilidad de mejorar el nivel académico de la población, si está en sus manos rediseñar estrategias de comunicación con un lenguaje más sencillo capaz de impactar a mujeres de bajo nivel educativo.

El estado civil también tuvo un importante rol en la práctica de estos exámenes, el 
hecho de estar casada se asoció de manera positiva con la práctica de la mamografía, ya que se observó que las mujeres casadas asisten a realizarse en mayor proporción la prueba. (Ávila I, 2013), (Vázquez M, 2004).

La historia familiar de cáncer de mama está relacionada con la percepción de riesgo 0 susceptibilidad de desarrollar cáncer de mama, más si está influenciada por parte del personal de salud frente familiares de pacientes con cáncer (Lee-Lin $F$, 2007). Esto explicaría su relación con la práctica sobre todo del ECM.

Una publicación reciente plantea una característica genética como factor protector para cáncer de mama en mujeres con raíces latinoamericanas. (Ferjerman, 2014). De confirmarse este descubrimiento esto podría influir en la práctica de pruebas de detección temprana ya que se determinaría con una prueba genética un determinado grupo de mujeres en riesgo de contraer cáncer de mama.

Las mujeres ubicadas en zonas urbanas tuvieron una mayor asociación con la práctica de estas pruebas, quizás en relación a un mayor acceso a los servicios de salud. Medellín seguida de Bogotá y Cali fueron las ciudades que reportaron mayor cobertura de mamografía (55\%, 54\%, 49\% respectivamente). (ENDS 2010).

El índice de riqueza también estuvo asociado con la práctica de estas pruebas, esto puede deberse a que con un mayor número de ingreso es mucho más fácil hacer frente a todo lo relacionado con afrontar los costos de tratamiento y rehabilitación de la enfermedad. Esta situación se ratifica al asociar el uso de la mamografía con factores como el trabajar y contar con pensión lo que se ve reflejado a un mayor acceso a los servicios de salud.

En relación a los factores asociados al ámbito personal y a los estilos de vida, llama la atención el tamaño y número de hijos ya que las mujeres que conviven con menos de 4 personas tienen una mayor utilización de la mamografía. Andersen \&Laake, 1983en Noruega, encontraron que después de ajustar para otras variables como necesidad en salud, una familia grande determinó una menor utilización.

La dieta juega un papel determinante en la prevención del cáncer, en general el consumo de frutas y verduras sumadas a la práctica de exámenes de detección temprana, pueden contribuir a diferencias étnicas y raciales en las tasas de aparición del cáncer de mama. (Gorin SS. 2001). La asociación entre el consumo de frutas y verduras y la práctica de la mamografía es atribuible al autocuidado. En este mismo sentido se puede entender la presencia de comorbilidades con la práctica de la mamografía, ya que la existencia de enfermedades crónicas implica un mayor contacto con los servicios de salud.(Perea M Dolores 2012).Sin embargo JiménezGarcía et al presentaron resultados en sentido contrario en mujeres con diabetes.

El conocimiento del cáncer de mama como una enfermedad relacionada con el sufrimiento, la muerte, la pérdida del entorno familiar, la perdida de la autoestima, sin mencionar el alto costo que esta conlleva hace que en muchos casos la mujer se concientice de la importancia de la práctica de pruebas que permitan su detección en estadios tempranos lo que beneficiaria su tratamiento y calidad de vida en caso de presentar esta enfermedad. Sin embargo el entorno cultural y los mitos presentados no solo en la población sino también desde la parte médica muchas veces limitan el acceso de las mujeres a este tipo de pruebas.

Los estudios anteriormente citados no mencionan de manera precisa las barreras administrativas como factores determinantes en la no practica del ECM y la mamografía, sin embargo las bajas coberturas para estas pruebas denotan que existen falencias por parte de los sistemas de salud en cuanto a la sensibilización, y capacitación del personal de salud para 
motivar e incentivar las mujeres en la búsqueda de un diagnóstico oportuno. Las restricciones al personal de la salud en términos de tiempo y de solicitud de exámenes, también inciden de manera negativa en la práctica de estas pruebas. Las barreras para el acceso oportuno a pruebas de tamización y diagnósticas y finalmente la carencia por parte de las IPS de protocolos o guías de manejo de la patología mamaria, hacen que cada vez sea más difícil el abordaje en términos de detección temprana del cáncer de mama.

Para hacer frente a esta problemática se hace necesario enfrentar aquellos factores modificables desde el ámbito administrativo y personal relacionado con los estilos vida. Y la manera más adecuada es desde la educación, porque no sería suficiente contar con programas de tamización bien estructurados si no existe una sensibilización por parte de las usuarias de estos programas, además de la educación al personal de salud no solo desde la parte asistencial sino también administrativa; la sensibilización debe hacerse de carácter urgente de modo centralizado a las empresas prestadoras de salud comprometiéndolas de manera obligatoria y con sanciones al no cumplimiento de las actividades relacionadas con la detección temprana del cáncer.

\section{REFERENCIAS BIBLIOGRAFICAS}

Alexander FE, Anderson TJ, Brown HK, Forrest AP, Hepburn W, Kirkpatrick AE et al. (1999) . 14 years follow up from the Edinburgh randomized trials of breast cancer screening. Lancet; 1903-8.

Anderson BO, Braun S, Lim S, Smith RA, TaplinS, Thomas DB. (2003). Global Summit Early Detection Panel.Early detection of breast cancer in countries with limited resources. Breast J,9(Suppl.2):S51-9.
Andersson I JL. (1997). Reduced breast cancer mortality in women under age 50: update results from the Malmo Mammographic Screening Program. J Natl Cancer Inst;63-7.

Andersen AS, Laake P. (1983) A causal model for physician utilization: analysis of Norwegian data. MedCare; 21(3):266-78.

Arboleda W, Sánchez O, Poveda C, Casas C, Rincón L. (2009). Cobertura de examen clínico y mamografía de tamización para cáncer de mama en mujeres bogotanas and Mammography Amonginsured women in Bogotá. 13(9):69-76.

Arboleda W, Betancourt D, Correa L.(2012). Frecuencia de Mamografía y Examen Clínico de Mama en mujeres del régimen subsidiado- Manizales (Caldas). Hacia la Promoción de la Salud, Volumen 17, No.2, julio, págs. $125-135$.

Ávila I, Bello L, Martelo L, Moscote G, Orozco W, Velasco E, Visbal M. (2013) Factores asociados al uso de mamografía en mujeres mayores de 50 años. Cartagena. Rev. Cienc. Salud. 12 (2): 183-193.

Carvalho A, Olivera M, Ferreira E. (2006). Práctica del autoexamen de mamas por usuarias del sistema único de salud de Ceará. Rev Cubana Enfermer ;22(3).

D. Kardinah, Benjamin O. Anderson, Catherine Duggan, Ibrahim A Ali and David B Thomas. (2014). Short report: Limited effectiveness of screening mammography in addition to clinical breast examination by trained nurse midwives in rural Jakarta, Indonesia. Int. J. Cancer: 134, 1250-1255

Díaz S. Piñeros M, Sánchez O. (2005) Detección temprana del cáncer de mama: aspectos críticos para un programa de tamizaje organizado en Colombia Early detection of breast cancer : critical aspects for an organized screening programme in Colombia.(528):93-105. 
Fatema I. Boxwala $\mathbb{E}$ Areeta BridgemohanÆEDerek M. Griffith $\mathbb{E}$ Amr S. Soliman.Factors Associated with Breast Cancer Screening in Asian Indian Women in Metro-Detroit. J Immigrant Minority Health. DOI 10.1007/s10903-009-9277-0

Fejerman L, Amadiyeh N, Hu D, Huntsman S, Beckman K B. (2 Genome-wide association study of breast cancer in Latinas identifies novel protective variants on 6q25014). Nature Communications 5, Article number: 5260 doi:10.1038/ncomms6260

Ferlay J, Soerjomataram I, Ervik M, Dikshit R, Eser S, Mathers C, Rebelo M,Parkin DM, Forman D, Bray, F.GLOBOCAN 2012 v1.0, Cancer Incidence and Mortality Worldwide: IARC Cancer Base No. 11 [Internet].Lyon, France: International Agency for Research on Cancer; 2013. Available from: http://globocan.iarc.fr, accessed on day15/jun/2014.

Frisell J. (1997). Lidbrink E HL. Follow after 11 years- update of mortality results in the Stockholm mammographic screening trials. Breast Cancer Res Treat;45:263-70.

Gorin SS, Jacobson J. (2001). Diet and breast cancer surveillance behaviors among Harlem women. Ann N Y AcadSci ; 952:15360.

Humphrey LL, Helfan M, Chan B WS. (2002).Breast cancer screening: a summary of the evidence for the US preventive services Task Force. Ann Intern Med [Internet]; 137:347-60. Available from: Disponible<www.guideline.gov].

IARC Handbooks of Cancer Prevention Volume 7.International Agency for Research on Cancer. Breast Cancer Screening 2002., Lyon: IARC Press; 2002.

Jiménez-García R. (2009). HernándezBarrera V, Carrasco-Garrido $P$, et al. Prevalence and predictors of breast and cervical cancer screening among Spanish women with diabetes. Diabetes Care; 32:1470-2.

Kosters JP (2004).Regular self-examination or clinical examination for early detection of breast cancer.Cochrane Libr. (Issue 1)

Lee IC, Lehman CD. (2013).Tomosintesis de mama digital y los desafíos de la implementación de una tecnología de detección temprana de mama en la práctica clínica. PubMed J AmColl Radiol. 2013 Dic, 10 (12):913-7.

Lee-Lin F. (2007). Menon U, Pett M, Nail L, Lee S, Mooney K.Breast cancer beliefs and mammography screening practices among Chinese American immigrants. J Obstet Gynecol Neonatal Nurs ;36(3):212-21.

López L, Suárez-López L, Torres-Sánchez L. (2009).Detección del cáncer de mama en México: síntesis de los resultados de la Encuesta Nacional de Salud Reproductiva. Salud Publica Mex; 51(supl 2):345-9.

Manrique F, Ospina J, Vega N, Morales A, Amaya G. (2012). Factores asociados a la práctica correcta del autoexamen de mama en mujeres de Tunja (Colombia). Invest Educ Enferm; 30(1).

Martín-López R, Hernández-Barrera V, De Andrés AL, et al. (2010). Breast and cervical cancer screening in Spain and predictors of adherence.Eur J Cancer Prev; 19:239-45.

Miller A, Wall C, Baines C, To T, Narod S. (2014). Twenty five year follow-up for breast cancer incidence and mortality of the Canadian National Breast Screening Study: randomised screening trial. BMJ; 348:g366doi: 10.1136/bmj.g366.

Miller AB. (2008).Practical applications for clinical breast examination (CBE) and breast self-Short Report1254 Limited effectiveness of screening mammography examination (BSE) in screening and early detection of 
breast cancer. Breast care (Basel, Switzerland); 3:17-20.

NS. W. (2003). Breast cancer mortality in relation to clinical breast examination and breast and breast self-examination. Breast J: 86-9.

Olsen O. (2004). GP.Screening for breast cancer with mammography. Cochrane Libr;(I).

Perea M Dolores (2012). Vinyals G, Altzibar $\mathrm{J}$, Ascunce N, Moreno V. Cancer screening practices and associated lifestyles in population controls of the Spanish multicase control study. Gac Sanit vol.26 n.4 Barcelona.

Prendes Marianela, Soler V. C, Morales W,. (1998).Pesquisaje de Cáncer de mama en la comunidad de Santos Suárez. Rev. Cubana Med Gen Integr; 14(2):165-70.

República de Colombia, Ministerio de la Protección Social, Instituto Nacional de Cancerología (2010). Atlas de Mortalidad por cáncer en Colombia. Bogotá : Piñeros $\mathrm{M}$, Pardo C.

República de Colombia, Ministerio de Protección social (2000). Resolución 0412: Norma técnica de detección temprana de cáncer de mama.

República de Colombia, Ministerio de Protección Social (2000). Resolución 3384, por la cual se modifica parcialmente la resolución 412 y 1745 del año 2000.

República de Colombia, Comisión de Regulación en Salud (2011). Acuerdo No 029, por el cual se sustituye el acuerdo 028 de 2011 que define, aclara y actualiza integralmente el Plan Obligatorio de Salud.

República de Colombia Instituto Nacional de Cancerología (2006). Recomendaciones para la tamización y la detección temprana del cáncer de mama en Colombia. Bogotá.
República de Colombia, Congreso de Colombia (2010). Ley 1384 Sandra Ceballos, por la cual se establecen las acciones para la atención integral del Cáncer en Colombia.

República de Colombia, Ministerio de Salud y Protección Social, Instituto Nacional de Cancerología ESE (2012). Plan Decenal para el Control del Cáncer en Colombia, 2012 - 2021.

República de Colombia (2010), Ministerio de la protección Social, Profamilia. Encuesta Nacional de demografía y salud. ENDS 2010 [Internet]. Profamilia. 2010 [cited 2014 SEP 21]. p. 439-75. Available from: http://www.profamilia.org.co.

República de Colombia (2012), Ministerio de Salud y Protección Social, Instituto Nacional de Cancerología. Manual Para La Detección del Cáncer de Mama. Available from http//www. cancer.gov.co.

República de Colombia. (2013). Ministerio de Salud y Protección Social. Guía de práctica clínica para la detección temprana, tratamiento integral, seguimiento, y Rehabilitación del cáncer de Mama.

República de Colombia. (2005). Ministerio de la Protección Social, Profamilia. Encuesta Nacional de Demografía y Salud. Bogotá.

Semiglazov VF. (1993). Sagadai VN MV. Study of the role of breast self - examination in the reduction of mortality from breast cancer. Eur J cancer.2039-46.

Shapiro S. (1997) Periodic screening for breast cancer: the HIP randomized controlled trial health insurance plan. J Natl Cancer Inst Monog; 27-30.

Sankaranarayanan R, Ramadas K, Thara S, Muwonge R, Prabhakar J, Augustine P, et al. (2011). Clinical breast examination: preliminary results from a cluster 
randomized controlled trial in India. J. Natl. Cancer Inst.

Tabar L, Vitak B, Chen HH, Duffy Sw, Yen MF, Chiang CF et al.(2000) The Swedinsh two/county trial twenty years later. Update mortality results and new insights from long/term follow up. Radiol Clin North Am; 625-51.

Tabár L, Vitak B, Chen TH, Yen AM, Cohen A, Tot T, Chiu SY, Chen SL, Fann JC, Rosell J, Fohlin H, Smith RA, Duffy SW.(2011). Swedish two-county trial: impact of mammographic screening on breast cancer mortality during 3 decades. Radiology. 2011 Sep;260(3):658-63. doi: 10.1148/radiol.11110469. Epub 2011 Jun 28.

Thomas D, Gao D, Ray M, Ray R, Wang W, Allison Ch et al. (2002) Randomized trial of breast self-examination in shangai. J Natl Cancer Inst. 1445-57.

Vázquez M.(2004). Uso de acciones preventivas por la población de María Tamaulipas. [tesis maestría Ciencia de Enfermería]. [San Nicolás de los Garza]: Universidad Autónoma de Nuevo León. 33 p.

Villacrés K. (2002). Alarcón AF. Estudio Piloto sobre actitudes preventivas para cáncer de mama en Mujeres de Sullana. Rev Med Hered; 13 (4):131-4.

Wasileh Petro-Nustus, Sc.D., R.N., and Blanche I. Mikhail, D NSc, R.N., C.N.S.(2002).Factors Associated with Breast Self-Examination Among Jordanian Women public Health Nursing Vol.19 No.4, pp.263-271. 